\title{
A family of pseudorandom binary sequences constructed by the multiplicative inverse
}

by

HuAning LiU (Xi'an)

1. Introduction. Let $p$ be an odd prime. For each integer $a$ with $t<$ $a \leq t+u$ and $(a, p)=1$, there exists one and only one $\bar{a}$ such that $0<\bar{a}<p$ and $a \bar{a} \equiv 1(\bmod p)$. Let $r(p, u, t)$ be the number of cases in which $a$ and $\bar{a}$ are of opposite parity, that is

$$
r(p, u, t)=\sum_{\substack{t<a \leq t+u \\(a, p)=1 \\ 2 \nmid a+\bar{a}}} 1
$$

Define

$$
E(p, u, t)=r(p, u, t)-\frac{1}{2} \sum_{\substack{t<a \leq t+u \\(a, p)=1}} 1 \quad \text { and } \quad S(p, u)=\sum_{t=1}^{p}|E(p, u, t)|^{2} .
$$

W. Zhang [14] showed that

$$
S(p, u)=\frac{1}{4} u p+O\left(u^{2} \sqrt{p} \log ^{2} p\right)
$$

by proving the estimate

$$
\sum_{\substack{n=1 \\ p \nmid n+x}}^{p-1}(-1)^{\bar{n}+\overline{n+x}} \ll \sqrt{p} \log ^{2} p .
$$

Therefore it is natural to expect that the sequence $\left\{(-1)^{\bar{n}+\overline{n+x}}\right\}$ behaves like a random sequence of \pm signs.

2000 Mathematics Subject Classification: Primary 11K45.

Key words and phrases: pseudorandom binary sequence, multiplicative inverse, exponential sum.

Supported by the National Natural Science Foundation of China under Grant No. 60472068 and No. 10671155; Natural Science Foundation of Shaanxi province of China under Grant No. 2006A04; and the Natural Science Foundation of the Education Department of Shaanxi Province of China under Grant No. 06JK168. 
In a series of papers C. Mauduit, J. Rivat and A. Sárközy (partly with other coauthors) studied finite pseudorandom binary sequences

$$
E_{N}=\left\{e_{1}, \ldots, e_{N}\right\} \in\{-1,+1\}^{N} .
$$

In [9] C. Mauduit and A. Sárközy first introduced the following measures of pseudorandomness: the well-distribution measure of $E_{N}$ is defined by

$$
W\left(E_{N}\right)=\max _{a, b, t}\left|\sum_{j=0}^{t-1} e_{a+j b}\right|,
$$

where the maximum is taken over all $a, b, t \in \mathbb{N}$ with $1 \leq a \leq a+(t-1) b \leq N$; the correlation measure of order $k$ of $E_{N}$ is

$$
C_{k}\left(E_{N}\right)=\max _{M, D}\left|\sum_{n=1}^{M} e_{n+d_{1}} \cdots e_{n+d_{k}}\right|,
$$

where the maximum is taken over all $D=\left(d_{1}, \ldots, d_{k}\right)$ and $M$ with $0 \leq$ $d_{1}<\cdots<d_{k} \leq N-M$; and the combined (well-distribution-correlation) PR-measure of order $k$,

$$
Q_{k}\left(E_{N}\right)=\max _{a, b, t, D}\left|\sum_{j=0}^{t} e_{a+j b+d_{1}} \cdots e_{a+j b+d_{k}}\right|,
$$

is defined for all $a, b, t, D=\left(d_{1}, \ldots, d_{k}\right)$ with $1 \leq a+j b+d_{i} \leq N$ $(i=1, \ldots, k)$. In [10] the connection between the measures $W$ and $C_{2}$ was studied.

The sequence $E_{N}$ is considered to be a "good" pseudorandom sequence if both $W\left(E_{N}\right)$ and $C_{k}\left(E_{N}\right)$ (at least for small $k$ ) are "small" in terms of $N$. Later J. Cassaigne, C. Mauduit and A. Sárközy [3] proved that this terminology is justified since for almost all $E_{N} \in\{-1,+1\}^{N}$, both $W\left(E_{N}\right)$ and $C_{k}\left(E_{N}\right)$ are less than $N^{1 / 2} \log ^{c} N$.

It was shown in [9] that the Legendre symbol forms a good pseudorandom sequence. In [1] and [2], J. Cassaigne and coauthors studied the pseudorandomness of the Liouville function, defined as $\lambda(n)=(-1)^{\Omega(n)}$ $(\Omega(n)=$ the number of prime factors of $n$ counted with multiplicity) and also of $\gamma(n)=(-1)^{\omega(n)}(\omega(n)=$ the number of distinct prime factors of $n)$. Moreover, let

$$
K(m, n ; p)=\sum_{a=1}^{p-1} e\left(\frac{m a+n \bar{a}}{p}\right)
$$

denote the Kloosterman sums, where $e(y)=e^{2 \pi i y}, p$ is a prime, and $\bar{a}$ is the multiplicative inverse of $a$ modulo $p$ such that $1 \leq \bar{a} \leq p-1$. E. Fouvry (with coauthors) [4] showed that the signs of $K(1, n ; p)$ form a good pseudorandom binary sequence. 
Furthermore, let $p$ be an odd prime, and $g$ a primitive root modulo $p$. Define ind $n$ by $1 \leq$ ind $n \leq p-1$ and $n \equiv g^{\text {ind } n}(\bmod p)$. Write $N=p-1$ and define the sequence $E_{N}=\left\{e_{1}, \ldots, e_{N}\right\}$ by

$$
e_{n}= \begin{cases}+1 & \text { if } 1 \leq \text { ind } n \leq(p-1) / 2 \\ -1 & \text { if }(p+1) / 2 \leq \text { ind } n \leq p-1 .\end{cases}
$$

A. Sárközy [13] showed that $E_{N}$ is also a good pseudorandom binary sequence.

However, the above constructions produce only a few good sequences while in certain applications (e.g., in cryptography) one needs large families of good pseudorandom binary sequence. Therefore some large families of pseudorandom binary sequences were introduced in [5], [6], [8] and [11].

As was said in [9], the analysis of the known constructions leads to the conclusion that, although the new constructions are superior to the previous ones from many points of view, there is a price paid for this so that there is no perfect construction. Thus the selection of the construction method to be applied must depend on the application in mind; the construction which is superior in a certain situation may fail in another one. This also means that the search for new approaches and new constructions should be continued.

Let $p$ be an odd prime. Define

$$
e_{n}^{\prime}= \begin{cases}(-1)^{\bar{n}+\overline{n+x}} & \text { if } p \nmid n \text { and } p \nmid n+x, \\ 1 & \text { otherwise, }\end{cases}
$$

where $x$ is an integer with $1 \leq x \leq p-1$. Let $E_{p-1}^{\prime}=\left\{e_{1}^{\prime}, \ldots, e_{p-1}^{\prime}\right\}$ be defined by (1.1). In [7] we proved that

$$
\begin{aligned}
& W\left(E_{p-1}^{\prime}\right) \ll p^{1 / 2} \log ^{3} p, \\
& C_{2}\left(E_{p-1}^{\prime}\right) \ll p^{1 / 2} \log ^{5} p, \\
& Q_{2}\left(E_{p-1}^{\prime}\right) \ll p^{1 / 2} \log ^{5} p .
\end{aligned}
$$

This shows that $\left\{(-1)^{\bar{n}+\overline{n+x}}\right\}$ is a good pseudorandom binary sequence.

However, it is usually not enough to control correlations of order 2 to ensure the pseudorandom behavior of a sequence, in particular in the case of applications to cryptography. Therefore in the report for our paper [7] the referee suggested completing that study by showing analogous results for correlations of larger order, $C_{k}$ and $Q_{k}$ for $k>2$. Moreover, he/she suggested completing that work by studying the measure of pseudorandomness for the more general construction obtained by

$$
e_{n}^{\prime \prime}= \begin{cases}(-1)^{\overline{f(n)}+\overline{f(n+x)}} & \text { if } p \nmid f(n) \text { and } p \nmid f(n+x), \\ 1 & \text { otherwise, }\end{cases}
$$

where $f$ is a suitable polynomial over $\mathbb{F}_{p}$. 
In this paper, we realize the referee's suggestions. The main results are the following.

Theorem 1.1. Let $p$ be an odd prime, and let $E_{p-1}^{\prime}=\left\{e_{1}^{\prime}, \ldots, e_{p-1}^{\prime}\right\}$ be defined by (1.1). Then

$$
C_{k}\left(E_{p-1}^{\prime}\right) \ll k p^{1 / 2} \log ^{2 k+1} p, \quad Q_{k}\left(E_{p-1}^{\prime}\right) \ll k p^{1 / 2} \log ^{2 k+1} p .
$$

Theorem 1.2. Let $p$ be an odd prime, and let $f(x) \in \mathbb{F}_{p}[x]$ have degree $d$ with $0<d<p$ and no multiple zero in $\overline{\mathbb{F}}_{p}$. Let $E_{p-1}^{\prime \prime}=\left\{e_{1}^{\prime \prime}, \ldots, e_{p-1}^{\prime \prime}\right\}$ be defined by (1.2). Assume that $k \in \mathbb{N}$ with $2 \leq k \leq p$, and one of the following conditions holds:

$$
\text { (i) } \quad k=2 ; \quad \text { (ii) } \quad(4 d)^{k}<p \text {. }
$$

Then

$$
\begin{aligned}
& W\left(E_{p-1}^{\prime \prime}\right) \ll d p^{1 / 2} \log ^{3} p, \\
& C_{k}\left(E_{p-1}^{\prime \prime}\right) \ll k d p^{1 / 2} \log ^{2 k+1} p, \\
& Q_{k}\left(E_{p-1}^{\prime \prime}\right) \ll k d p^{1 / 2} \log ^{2 k+1} p .
\end{aligned}
$$

REMARK. Since there is a very good (polynomial time) algorithm for computing the multiplicative inverse modulo $p$, these two sequences can be generated fast.

2. Some lemmas. To prove the theorems, we need the following lemmas.

Lemma $2.1([12])$. Let $g(x), h(x) \in \mathbb{F}_{p}[x]$ be such that the rational function $f(x)=g(x) / h(x)$ is not constant on $\mathbb{F}_{p}$, and let $s$ be the number of distinct roots of $h(x)$. Then

$$
\left|\sum_{\substack{n \in \mathbb{F}_{p} \\ h(n) \neq 0}} e\left(\frac{g(n)}{h(n) p}\right)\right| \leq(\max (\operatorname{deg}(g), \operatorname{deg}(h))+s-1) \sqrt{p} .
$$

Lemma 2.2. For any integers $s_{1}, \ldots, s_{l}, d_{1}, \ldots, d_{l}$ with $\left(s_{1} \cdots s_{l}, p\right)=1$ and $d_{1}<\cdots<d_{l}$, the polynomial

$$
\Omega_{1}(n):=\sum_{i=1}^{l} s_{i} \prod_{\substack{j=1 \\ j \neq i}}^{l}\left(n+d_{j}\right)
$$

is not the zero polynomial on $\mathbb{F}_{p}$. 
Proof. Suppose that $\Omega_{1}(n) \equiv 0(\bmod p)$. Then the coefficients of $n^{l-1}, \ldots, n, n^{0}$ must be congruent to 0 modulo $p$. So we have

$$
\sum_{j=1}^{l} s_{j}\left(\sum_{\substack{1 \leq i_{1}<\cdots<i_{k} \leq l \\ i_{1}, \ldots, i_{k} \neq j}} d_{i_{1}} \cdots d_{i_{k}}\right) \equiv 0(\bmod p), \quad k=0,1, \ldots, l-1 .
$$

This gives

$$
\begin{array}{r}
\sum_{j=1}^{l} s_{j}\left(\sum_{m=1}^{k+1}(-1)^{m-1} d_{j}^{m-1} \sum_{\substack{1 \leq i_{m}<\cdots<i_{k} \leq l \\
m \leq k}} d_{i_{m}} \cdots d_{i_{k}}\right) \equiv 0(\bmod p), \\
k=0,1, \ldots, l-1 .
\end{array}
$$

Substitute the $k$ th equation into the $(k+1)$ st equation for $k=0,1, \ldots, l-2$. Then we have

$$
\left\{\begin{array}{l}
s_{1}+s_{2}+\cdots+s_{l} \equiv 0(\bmod p) \\
s_{1} d_{1}+s_{2} d_{2}+\cdots+s_{l} d_{l} \equiv 0(\bmod p) \\
s_{1} d_{1}^{2}+s_{2} d_{2}^{2}+\cdots+s_{l} d_{l}^{2} \equiv 0(\bmod p) \\
\cdots \cdots \cdots \cdots \cdots \cdots \cdots \cdots \cdots \cdots \cdots \cdots \cdots+s_{l} d_{l}^{l-2} \equiv 0(\bmod p) \\
s_{1} d_{1}^{l-2}+s_{2} d_{2}^{l-2}+\cdots \cdots(\bmod p) . \\
s_{1} d_{1}^{l-1}+s_{2} d_{2}^{l-1}+\cdots+s_{l} d_{l}^{l-1} \equiv 0 .
\end{array}\right.
$$

That is,

$$
\left[\begin{array}{llll}
1 & 1 & \cdots & 1 \\
d_{1} & d_{2} & \cdots & d_{l} \\
\vdots & \vdots & & \vdots \\
d_{1}^{l-1} & d_{2}^{l-1} & \cdots & d_{l}^{l-1}
\end{array}\right]\left[\begin{array}{l}
s_{1} \\
s_{2} \\
\vdots \\
s_{l}
\end{array}\right] \equiv\left[\begin{array}{l}
0 \\
0 \\
\vdots \\
0
\end{array}\right](\bmod p)
$$

Since

$$
\left|\begin{array}{llll}
1 & 1 & \cdots & 1 \\
d_{1} & d_{2} & \cdots & d_{l} \\
\vdots & \vdots & & \vdots \\
d_{1}^{l-1} & d_{2}^{l-1} & \cdots & d_{l}^{l-1}
\end{array}\right|=\prod_{1 \leq j<i \leq l}\left(d_{i}-d_{j}\right) \neq 0
$$

(2.1) has one and only one solution

$$
\left[\begin{array}{l}
s_{1} \\
s_{2} \\
\vdots \\
s_{l}
\end{array}\right] \equiv\left[\begin{array}{l}
0 \\
0 \\
\vdots \\
0
\end{array}\right](\bmod p)
$$


which is impossible. This shows that $\Omega_{1}(n)$ is not the zero polynomial on $\mathbb{F}_{p}$.

Lemma 2.3. For any integers $a, b, u, x, d_{1}, \ldots, d_{k}, r_{1}, \ldots, r_{k}, s_{1}, \ldots, s_{k}$ such that $d_{1}<\cdots<d_{k}$ and $\left(b x r_{1} \cdots r_{k} s_{1} \cdots s_{k}, p\right)=1$,

$$
\begin{aligned}
\Psi_{1}:= & \sum_{\substack{j=0 \\
p \nmid\left(a+j b+d_{1}\right) \cdots\left(a+j b+d_{k}\right) \\
p \nmid\left(a+j b+d_{1}+x\right) \cdots\left(a+j b+d_{k}+x\right)}}^{p-1} e\left(\frac{r_{1} \overline{a+j b+d_{1}}+\cdots+r_{k} \overline{a+j b+d_{k}}}{p}\right) \\
& \times e\left(\frac{s_{1} \overline{a+j b+d_{1}+x}+\cdots+s_{k} \overline{a+j b+d_{k}+x}+u j}{p}\right) \\
& \ll k \sqrt{p} .
\end{aligned}
$$

Proof. From the properties of residue systems we have

$$
\begin{aligned}
\Psi_{1}= & \sum_{\substack{j=0 \\
p \nmid\left(j+d_{1}\right) \cdots\left(j+d_{k}\right) \\
p \nmid\left(j+d_{1}+x\right) \cdots\left(j+d_{k}+x\right)}}^{p-1} e\left(\frac{r_{1} \overline{j+d_{1}}+\cdots+r_{k} \overline{j+d_{k}}}{p}\right) \\
\times & e\left(\frac{s_{1} \overline{j+d_{1}+x}+\cdots+s_{k} \overline{j+d_{k}+x}+u \bar{b}(j-a)}{p}\right) .
\end{aligned}
$$

If $p \nmid u$, define

$$
H_{1}(j)=\left(j+d_{1}\right) \cdots\left(j+d_{k}\right)\left(j+d_{1}+x\right) \cdots\left(j+d_{k}+x\right)
$$

and

$$
\begin{aligned}
G_{1}(j)= & r_{1}\left(j+d_{2}\right) \cdots\left(j+d_{k}\right)\left(j+d_{1}+x\right) \cdots\left(j+d_{k}+x\right)+\cdots \\
& +r_{k}\left(j+d_{1}\right) \cdots\left(j+d_{k-1}\right)\left(j+d_{1}+x\right) \cdots\left(j+d_{k}+x\right) \\
& +s_{1}\left(j+d_{1}\right) \cdots\left(j+d_{k}\right)\left(j+d_{2}+x\right) \cdots\left(j+d_{k}+x\right)+\cdots \\
& +s_{k}\left(j+d_{1}\right) \cdots\left(j+d_{k}\right)\left(j+d_{1}+x\right) \cdots\left(j+d_{k-1}+x\right) \\
& +u \bar{b}(j-a)\left(j+d_{1}\right) \cdots\left(j+d_{k}\right)\left(j+d_{1}+x\right) \cdots\left(j+d_{k}+x\right) .
\end{aligned}
$$

The function $G_{1}(j)$ cannot be constant over $\mathbb{F}_{p}$ since the coefficient of $j^{2 k+1}$ is $u \bar{b}$. Thus by Lemma 2.1 we have $\Psi_{1} \ll k \sqrt{p}$.

For $p \mid u$, we have

$$
\begin{aligned}
& \Psi_{1}=\sum_{\substack{j=0 \\
p \nmid\left(j+d_{1}\right) \cdots\left(j+d_{k}\right) \\
p \nmid\left(j+d_{1}+x\right) \cdots\left(j+d_{k}+x\right)}}^{p-1} e\left(\frac{r_{1} \overline{j+d_{1}}+\cdots+r_{k} \overline{j+d_{k}}}{p}\right) \\
& \times e\left(\frac{s_{1} \overline{j+d_{1}+x}+\cdots+s_{k} \overline{j+d_{k}+x}}{p}\right) .
\end{aligned}
$$

Defining

$$
F_{1}(j)=r_{1} \overline{j+d_{1}}+\cdots+r_{k} \overline{j+d_{k}}+s_{1} \overline{j+d_{1}+x}+\cdots+s_{k} \overline{j+d_{k}+x},
$$


and $d_{k+1}=d_{1}+x, \ldots, d_{2 k}=d_{k}+x, r_{k+1}=s_{1}, \ldots, r_{2 k}=s_{k}$, we get

$$
F_{1}(j)=r_{1} \overline{j+d_{1}}+\cdots+r_{2 k} \overline{j+d_{2 k}} \text {. }
$$

If there are some $n, m$ with $n<m$ and $d_{n}=d_{m}$, then

$$
\begin{aligned}
F_{1}(j)= & r_{1} \overline{j+d_{1}}+\cdots+r_{n-1} \overline{j+d_{n-1}}+\left(r_{n}+r_{m}\right) \overline{j+d_{n}}+r_{n+1} \overline{j+d_{n+1}} \\
& +\cdots+r_{m-1} \overline{j+d_{m-1}}+r_{m+1} \overline{j+d_{m+1}}+\cdots+r_{2 k} \overline{j+d_{2 k}} .
\end{aligned}
$$

If $p \mid r_{n}+r_{m}$, we define

$$
\begin{aligned}
F_{1}^{\prime}(j)= & r_{1} \overline{j+d_{1}}+\cdots+r_{n-1} \overline{j+d_{n-1}}+r_{n+1} \overline{j+d_{n+1}}+\cdots \\
& +r_{m-1} \overline{j+d_{m-1}}+r_{m+1} \overline{j+d_{m+1}}+\cdots+r_{2 k} \overline{j+d_{2 k}},
\end{aligned}
$$

hence $F_{1}(j) \equiv F_{1}^{\prime}(j)(\bmod p)$. If $p \nmid r_{n}+r_{m}$, then set $F_{1}^{\prime}(j)=F_{1}(j)$.

For $F_{1}^{\prime}(j)$, if there still exist some $n^{\prime}, m^{\prime}$ such that $n^{\prime}<m^{\prime}$ and $d_{n^{\prime}}=d_{m^{\prime}}$, then we continue the above process. Since $d_{1}<\cdots<d_{k}, d_{k+1}<\cdots<d_{2 k}$ and $d_{1}<d_{k+1}$, we finally get some

$$
F_{1}^{*}(j)=t_{1} \overline{j+c_{1}}+t_{2} \overline{j+c_{2}}+\cdots+t_{l} \overline{j+c_{l}}
$$

with $\left(t_{1} \cdots t_{l}, p\right)=1, c_{1}<\cdots<c_{l}$ and $F_{1}(j) \equiv F_{1}^{*}(j)(\bmod p)$. Therefore

$$
\Psi_{1}=\sum_{\substack{j=0 \\ p \nmid\left(j+c_{1}\right) \cdots\left(j+c_{l}\right)}}^{p-1} e\left(\frac{F_{1}^{*}(j)}{p}\right) .
$$

Now defining

$$
H_{1}^{*}(n)=\left(n+c_{1}\right) \cdots\left(n+c_{l}\right) \quad \text { and } \quad G_{1}^{*}(n)=\sum_{i=1}^{l} t_{i} \prod_{\substack{j=1 \\ j \neq i}}^{l}\left(n+c_{j}\right),
$$

we have

$$
\Psi_{1}=\sum_{\substack{n \in \mathbb{F}_{p} \\ H_{1}^{*}(n) \neq 0}} e\left(\frac{G_{1}^{*}(n)}{H_{1}^{*}(n) p}\right) .
$$

By Lemma 2.2 we know that $G_{1}^{*}(n)$ is not the zero polynomial on $\mathbb{F}_{p}$. Note that $\operatorname{deg}\left(G_{1}^{*}\right)<\operatorname{deg}\left(H_{1}^{*}\right)$, so $G_{1}^{*}(n) / H_{1}^{*}(n)$ is not constant on $\mathbb{F}_{p}$. Then from Lemma 2.1 we also have $\Psi_{1} \ll l \sqrt{p} \ll k \sqrt{p}$.

Lemma 2.4. Define $p, f(x), d$ and $k$ as in Theorem 1.2. Then for any integers $l, d_{1}, \ldots, d_{l}, s_{1}, \ldots, s_{l}$ with $1 \leq l \leq k, d_{1}<\cdots<d_{l}$ and $\left(s_{1} \cdots s_{l}, p\right)=1$, the polynomial

$$
\Omega_{2}(n):=\sum_{i=1}^{l} s_{i} \prod_{\substack{j=1 \\ j \neq i}}^{l} f\left(n+d_{j}\right)
$$

is not the zero polynomial on $\mathbb{F}_{p}$.

Proof. This lemma can be easily deduced from Lemma 5 of [11]. 
Lemma 2.5. Define $p, f(x), d$ and $k$ as in Theorem 1.2. For any integers $a, b, u, x, d_{1}, \ldots, d_{k}, r_{1}, \ldots, r_{k}, s_{1}, \ldots, s_{k}$ such that $d_{1}<\cdots<d_{k}$ and $\left(b x r_{1} \cdots r_{k} s_{1} \cdots s_{k}, p\right)=1$, we have

$$
\begin{aligned}
\Psi_{2}:= & \sum_{\substack{j=0 \\
p \nmid f\left(a+j b+d_{1}\right) \cdots f\left(a+j b+d_{k}\right) \\
p \nmid f\left(a+j b+d_{1}+x\right) \cdots f\left(a+j b+d_{k}+x\right)}}^{p-1} e\left(\frac{r_{1} \overline{f\left(a+j b+d_{1}\right)}+\cdots+r_{k} \overline{f\left(a+j b+d_{k}\right)}}{p}\right) \\
& \times e\left(\frac{s_{1} \overline{f\left(a+j b+d_{1}+x\right)}+\cdots+s_{k} \overline{f\left(a+j b+d_{k}+x\right)}+u j}{p}\right) \\
& \ll k d \sqrt{p} .
\end{aligned}
$$

Proof. From the properties of residue systems we have

$$
\begin{aligned}
\Psi_{2}= & \sum_{\substack{j=0 \\
p \nmid f\left(j+d_{1}\right) \cdots f\left(j+d_{k}\right) \\
p \nmid f\left(j+d_{1}+x\right) \cdots f\left(j+d_{k}+x\right)}}^{p-1} e\left(\frac{r_{1} \overline{f\left(j+d_{1}\right)}+\cdots+r_{k} \overline{f\left(j+d_{k}\right)}}{p}\right) \\
& \times e\left(\frac{s_{1} \overline{f\left(j+d_{1}+x\right)}+\cdots+s_{k} \overline{f\left(j+d_{k}+x\right)}+u \bar{b}(j-a)}{p}\right) .
\end{aligned}
$$

If $p \nmid u$, define

and

$$
H_{2}(j)=f\left(j+d_{1}\right) \cdots f\left(j+d_{k}\right) f\left(j+d_{1}+x\right) \cdots f\left(j+d_{k}+x\right)
$$

$$
\begin{aligned}
G_{2}(j)= & r_{1} f\left(j+d_{2}\right) \cdots f\left(j+d_{k}\right) f\left(j+d_{1}+x\right) \cdots f\left(j+d_{k}+x\right)+\cdots \\
& +r_{k} f\left(j+d_{1}\right) \cdots f\left(j+d_{k-1}\right) f\left(j+d_{1}+x\right) \cdots f\left(j+d_{k}+x\right) \\
& +s_{1} f\left(j+d_{1}\right) \cdots f\left(j+d_{k}\right) f\left(j+d_{2}+x\right) \cdots f\left(j+d_{k}+x\right)+\cdots \\
& +s_{k} f\left(j+d_{1}\right) \cdots f\left(j+d_{k}\right) f\left(j+d_{1}+x\right) \cdots f\left(j+d_{k-1}+x\right) \\
& +u \bar{b}(j-a) f\left(j+d_{1}\right) \cdots f\left(j+d_{k}\right) f\left(j+d_{1}+x\right) \cdots f\left(j+d_{k}+x\right) .
\end{aligned}
$$

The function $G_{2}(j)$ cannot be constant over $\mathbb{F}_{p}$ since $p \nmid u \bar{b}$. Thus by Lemma 2.1 we have $\Psi_{2} \ll k d \sqrt{p}$.

For $p \mid u$, we have

$$
\begin{aligned}
& \Psi_{2}=\sum_{\substack{j=0 \\
p \nmid f\left(j+d_{1}\right) \cdots f\left(j+d_{k}\right) \\
p \nmid f\left(j+d_{1}+x\right) \cdots f\left(j+d_{k}+x\right)}} e\left(\frac{r_{1} \overline{f\left(j+d_{1}\right)}+\cdots+r_{k} \overline{f\left(j+d_{k}\right)}}{p}\right) \\
& \times e\left(\frac{s_{1} \overline{f\left(j+d_{1}+x\right)}+\cdots+s_{k} \overline{f\left(j+d_{k}+x\right)}}{p}\right) .
\end{aligned}
$$

Defining

$F_{2}(j)=r_{1} \overline{f\left(j+d_{1}\right)}+\cdots+r_{k} \overline{f\left(j+d_{k}\right)}+s_{1} \overline{f\left(j+d_{1}+x\right)}+\cdots+s_{k} \overline{f\left(j+d_{k}+x\right)}$, 
and $d_{k+1}=d_{1}+x, \ldots, d_{2 k}=d_{k}+x, r_{k+1}=s_{1}, \ldots, r_{2 k}=s_{k}$, we get

$$
F_{2}(j)=r_{1} \overline{f\left(j+d_{1}\right)}+\cdots+r_{2 k} \overline{f\left(j+d_{2 k}\right)} \text {. }
$$

If there are some $n, m$ with $n<m$ and $d_{n}=d_{m}$, then

$$
\begin{aligned}
F_{2}(j)= & r_{1} \overline{f\left(j+d_{1}\right)}+\cdots+r_{n-1} \overline{f\left(j+d_{n-1}\right)}+\left(r_{n}+r_{m}\right) \overline{f\left(j+d_{n}\right)} \\
& +r_{n+1} \overline{f\left(j+d_{n+1}\right)}+\cdots+r_{m-1} \overline{f\left(j+d_{m-1}\right)} \\
& +r_{m+1} \overline{f\left(j+d_{m+1}\right)}+\cdots+r_{2 k} \overline{f\left(j+d_{2 k}\right)} .
\end{aligned}
$$

If $p \mid r_{n}+r_{m}$, we define

$$
\begin{aligned}
F_{2}^{\prime}(j)= & r_{1} \overline{f\left(j+d_{1}\right)}+\cdots+r_{n-1} \overline{f\left(j+d_{n-1}\right)}+r_{n+1} \overline{f\left(j+d_{n+1}\right)}+\cdots \\
& +r_{m-1} \overline{f\left(j+d_{m-1}\right)}+r_{m+1} \overline{f\left(j+d_{m+1}\right)}+\cdots+r_{2 k} \overline{f\left(j+d_{2 k}\right)}
\end{aligned}
$$

hence $F_{2}(j) \equiv F_{2}^{\prime}(j)(\bmod p)$. If $p \nmid r_{n}+r_{m}$, then set $F_{2}^{\prime}(j)=F_{2}(j)$.

For $F_{2}^{\prime}(j)$, if there still exist some $n^{\prime}, m^{\prime}$ such that $n^{\prime}<m^{\prime}$ and $d_{n^{\prime}}=d_{m^{\prime}}$, then we continue the above process. Since $d_{1}<\cdots<d_{k}, d_{k+1}<\cdots<d_{2 k}$ and $d_{1}<d_{k+1}$, we finally get some

$$
F_{2}^{*}(j)=t_{1} \overline{f\left(j+c_{1}\right)}+t_{2} \overline{f\left(j+c_{2}\right)}+\cdots+t_{l} \overline{f\left(j+c_{l}\right)}
$$

with $\left(t_{1} \cdots t_{l}, p\right)=1, c_{1}<\cdots<c_{l}$ and $F_{2}(j) \equiv F_{2}^{*}(j)(\bmod p)$. Therefore

$$
\Psi_{2}=\sum_{\substack{j=0 \\ p \nmid\left(j+c_{1}\right) \cdots\left(j+c_{l}\right)}}^{p-1} e\left(\frac{F_{2}^{*}(j)}{p}\right) .
$$

Now defining

$$
H_{2}^{*}(n)=f\left(n+c_{1}\right) \cdots f\left(n+c_{l}\right) \quad \text { and } \quad G_{2}^{*}(n)=\sum_{i=1}^{l} t_{i} \prod_{\substack{j=1 \\ j \neq i}}^{l} f\left(n+c_{j}\right)
$$

we have

$$
\Psi_{2}=\sum_{\substack{n \in \mathbb{F}_{p} \\ H_{2}^{*}(n) \neq 0}} e\left(\frac{G_{2}^{*}(n)}{H_{2}^{*}(n) p}\right) .
$$

By Lemma 2.4 we know that $G_{2}^{*}(n)$ is not the zero polynomial on $\mathbb{F}_{p}$. Note that $\operatorname{deg}\left(G_{2}^{*}\right)<\operatorname{deg}\left(H_{2}^{*}\right)$, so $G_{2}^{*}(n) / H_{2}^{*}(n)$ is not constant on $\mathbb{F}_{p}$. Then from Lemma 2.1 we also have $\Psi_{2} \ll l d \sqrt{p} \ll k d \sqrt{p}$.

3. Proof of the theorems. First we prove Theorem 1.1. For $1 \leq$ $a+t b+d_{i} \leq p-1, i=1, \ldots, k, 0 \leq d_{1}<\cdots<d_{k}$, by (1.1) and the trigonometric identity

$$
\sum_{u=1}^{p} e\left(\frac{u n}{p}\right)= \begin{cases}p & \text { if } p \mid n \\ 0 & \text { if } p \nmid n,\end{cases}
$$


we have

$$
\begin{aligned}
& \sum_{j=0}^{t} e_{a+j b+d_{1}}^{\prime} \cdots e_{a+j b+d_{k}}^{\prime}
\end{aligned}
$$

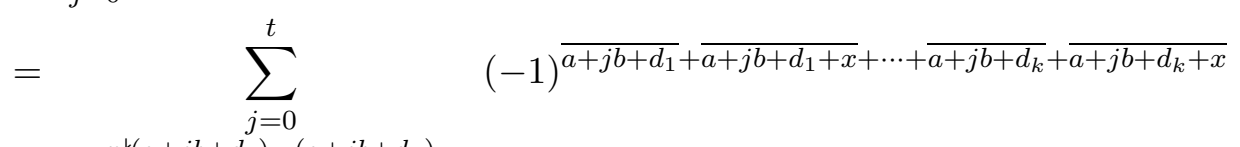

$$
\begin{aligned}
& p \nmid\left(a+j b+d_{1}\right) \cdots\left(a+j b+d_{k}\right) \\
& p \nmid\left(a+j b+d_{1}+x\right) \cdots\left(a+j b+d_{k}+x\right) \\
& +O(k) \\
& =\frac{1}{p^{2 k+1}} \quad \sum_{j=0}^{p-1} \quad \sum_{l=0}^{t} \sum_{u=1}^{p} e\left(\frac{u(j-l)}{p}\right) \\
& p \nmid\left(a+j b+d_{1}\right) \cdots\left(a+j b+d_{k}\right) \\
& p \nmid\left(a+j b+d_{1}+x\right) \cdots\left(a+j b+d_{k}+x\right) \\
& \times \sum_{m_{1}=1}^{p-1} \sum_{r_{1}=1}^{p} e\left(\frac{r_{1}\left(\overline{a+j b+d_{1}}-m_{1}\right)}{p}\right) \sum_{n_{1}=1}^{p-1} \sum_{s_{1}=1}^{p} e\left(\frac{s_{1}\left(\overline{a+j b+d_{1}+x}-n_{1}\right)}{p}\right) \\
& \times \cdots \times \sum_{m_{k}=1}^{p-1} \sum_{r_{k}=1}^{p} e\left(\frac{r_{k}\left(\overline{a+j b+d_{k}}-m_{k}\right)}{p}\right) \\
& \times \sum_{n_{k}=1}^{p-1} \sum_{s_{k}=1}^{p} e\left(\frac{s_{k}\left(\overline{a+j b+d_{k}+x}-n_{k}\right)}{p}\right)(-1)^{m_{1}+n_{1}+\cdots+m_{k}+n_{k}}+O(k) \\
& =\frac{1}{p^{2 k+1}} \sum_{r_{1}=1}^{p-1}\left(\sum_{m_{1}=1}^{p-1}(-1)^{m_{1}} e\left(-\frac{m_{1} r_{1}}{p}\right)\right) \sum_{s_{1}=1}^{p-1}\left(\sum_{n_{1}=1}^{p-1}(-1)^{n_{1}} e\left(-\frac{n_{1} s_{1}}{p}\right)\right) \\
& \times \cdots \times \sum_{r_{k}=1}^{p-1}\left(\sum_{m_{k}=1}^{p-1}(-1)^{m_{k}} e\left(-\frac{m_{k} r_{k}}{p}\right)\right) \sum_{s_{k}=1}^{p-1}\left(\sum_{n_{k}=1}^{p-1}(-1)^{n_{k}} e\left(-\frac{n_{k} s_{k}}{p}\right)\right) \\
& \times \sum_{u=1}^{p}\left(\sum_{l=0}^{t} e\left(-\frac{u l}{p}\right)\right) \\
& \times \quad \sum_{j=0}^{p-1} \quad e\left(\frac{r_{1} \overline{a+j b+d_{1}}+\cdots+r_{k} \overline{a+j b+d_{k}}}{p}\right) \\
& p \nmid\left(a+j b+d_{1}\right) \cdots\left(a+j b+d_{k}\right) \\
& p \nmid\left(a+j b+d_{1}+x\right) \cdots\left(a+j b+d_{k}+x\right) \\
& \times e\left(\frac{s_{1} \overline{a+j b+d_{1}+x}+\cdots+s_{k} \overline{a+j b+d_{k}+x}+u j}{p}\right) \\
& +O(k) \text {. }
\end{aligned}
$$


Since

$$
\begin{aligned}
& \sum_{l=0}^{t} e\left(-\frac{u l}{p}\right) \ll \frac{1}{|\sin (\pi u / p)|} \quad \text { for } p \nmid u, \\
& \sum_{m=1}^{p-1}(-1)^{m} e\left(-\frac{r m}{p}\right) \ll \frac{1}{|\sin (\pi / 2-\pi r / p)|},
\end{aligned}
$$

from Lemma 2.3 we have

$$
\begin{aligned}
\sum_{j=0}^{t} e_{a+j b+d_{1}}^{\prime} \cdots & e_{a+j b+d_{k}}^{\prime} \ll \frac{t}{p^{2 k+1}}\left(\sum_{r=1}^{p-1} \frac{1}{|\sin (\pi / 2-\pi r / p)|}\right)^{2 k} \cdot k \sqrt{p} \\
+ & \frac{1}{p^{2 k+1}}\left(\sum_{r=1}^{p-1} \frac{1}{|\sin (\pi / 2-\pi r / p)|}\right)^{2 k}\left(\sum_{u=1}^{p-1} \frac{1}{|\sin (\pi u / p)|}\right) \cdot k \sqrt{p} \\
& \ll k p^{1 / 2} \log ^{2 k+1} p .
\end{aligned}
$$

Therefore

$$
Q_{k}\left(E_{p-1}^{\prime}\right)=\max _{a, b, t, D}\left|\sum_{j=0}^{t} e_{a+j b+d_{1}}^{\prime} \cdots e_{a+j b+d_{k}}^{\prime}\right| \ll k p^{1 / 2} \log ^{2 k+1} p .
$$

Taking $a=0, b=1, j=n-1$ and $t=M-1$ in (3.3), we immediately get

$$
C_{k}\left(E_{p-1}^{\prime}\right)=\max _{M, D}\left|\sum_{n=1}^{M} e_{n+d_{1}}^{\prime} \cdots e_{n+d_{k}}^{\prime}\right| \ll k p^{1 / 2} \log ^{2 k+1} p .
$$

This proves Theorem 1.1.

Now we prove Theorem 1.2. For $1 \leq a+t b+d_{i} \leq p-1, i=1, \ldots, k$, $0 \leq d_{1}<\cdots<d_{k}$, by (1.2) and (3.1) we have

$$
\begin{aligned}
& \sum_{j=0}^{t} e_{a+j b+d_{1}}^{\prime \prime} \cdots e_{a+j b+d_{k}}^{\prime \prime} \\
& =\quad \sum_{j=0}^{t} \quad(-1)^{\overline{f\left(a+j b+d_{1}\right)}+\overline{f\left(a+j b+d_{1}+x\right)}+\cdots+\overline{f\left(a+j b+d_{k}\right)}} \\
& p \nmid f\left(a+j b+d_{1}\right) \cdots f\left(a+j b+d_{k}\right) \\
& p \nmid f\left(a+j b+d_{1}+x\right) \cdots f\left(a+j b+d_{k}+x\right) \\
& \times(-1)^{\overline{f\left(a+j b+d_{k}+x\right)}}+O(k d) \\
& =\frac{1}{p^{2 k+1}} \quad \sum_{j=0}^{p-1} \quad \sum_{l=0}^{t} \sum_{u=1}^{p} e\left(\frac{u(j-l)}{p}\right) \\
& p \nmid f\left(a+j b+d_{1}\right) \cdots f\left(a+j b+d_{k}\right) \\
& p \nmid f\left(a+j b+d_{1}+x\right) \cdots f\left(a+j b+d_{k}+x\right)
\end{aligned}
$$




$$
\begin{aligned}
& \times \sum_{m_{1}=1}^{p-1} \sum_{r_{1}=1}^{p} e\left(\frac{r_{1}\left(\overline{f\left(a+j b+d_{1}\right)}-m_{1}\right)}{p}\right) \\
& \times \sum_{n_{1}=1}^{p-1} \sum_{s_{1}=1}^{p} e\left(\frac{s_{1}\left(\overline{f\left(a+j b+d_{1}+x\right)}-n_{1}\right)}{p}\right) \\
& \times \cdots \times \sum_{m_{k}=1}^{p-1} \sum_{r_{k}=1}^{p} e\left(\frac{r_{k}\left(\overline{f\left(a+j b+d_{k}\right)}-m_{k}\right)}{p}\right) \\
& \times \sum_{n_{k}=1}^{p-1} \sum_{s_{k}=1}^{p} e\left(\frac{s_{k}\left(\overline{f\left(a+j b+d_{k}+x\right)}-n_{k}\right)}{p}\right)(-1)^{m_{1}+n_{1}+\cdots+m_{k}+n_{k}} \\
& +O(k d) \\
& =\frac{1}{p^{2 k+1}} \sum_{r_{1}=1}^{p-1}\left(\sum_{m_{1}=1}^{p-1}(-1)^{m_{1}} e\left(-\frac{m_{1} r_{1}}{p}\right)\right) \sum_{s_{1}=1}^{p-1}\left(\sum_{n_{1}=1}^{p-1}(-1)^{n_{1}} e\left(-\frac{n_{1} s_{1}}{p}\right)\right) \\
& \times \cdots \times \sum_{r_{k}=1}^{p-1}\left(\sum_{m_{k}=1}^{p-1}(-1)^{m_{k}} e\left(-\frac{m_{k} r_{k}}{p}\right)\right) \sum_{s_{k}=1}^{p-1}\left(\sum_{n_{k}=1}^{p-1}(-1)^{n_{k}} e\left(-\frac{n_{k} s_{k}}{p}\right)\right) \\
& \times \sum_{u=1}^{p}\left(\sum_{l=0}^{t} e\left(-\frac{u l}{p}\right)\right) \\
& \times \quad \sum_{j=0}^{p-1} \quad e\left(\frac{r_{1} \overline{f\left(a+j b+d_{1}\right)}+\cdots+r_{k} \overline{f\left(a+j b+d_{k}\right)}}{p}\right) \\
& p \nmid f\left(a+j b+d_{1}\right) \cdots f\left(a+j b+d_{k}\right) \\
& p \nmid f\left(a+j b+d_{1}+x\right) \cdots f\left(a+j b+d_{k}+x\right) \\
& \times e\left(\frac{s_{1} \overline{f\left(a+j b+d_{1}+x\right)}+\cdots+s_{k} \overline{f\left(a+j b+d_{k}+x\right)}+u j}{p}\right)+O(k d) .
\end{aligned}
$$

Then from (3.2) and Lemma 2.5 we get

$$
\begin{array}{r}
\sum_{j=0}^{t} e_{a+j b+d_{1}}^{\prime \prime} \cdots e_{a+j b+d_{k}}^{\prime \prime} \ll \frac{t}{p^{2 k+1}}\left(\sum_{r=1}^{p-1} \frac{1}{|\sin (\pi / 2-\pi r / p)|}\right)^{2 k} \cdot k d \sqrt{p} \\
+\frac{1}{p^{2 k+1}}\left(\sum_{r=1}^{p-1} \frac{1}{|\sin (\pi / 2-\pi r / p)|}\right)^{2 k}\left(\sum_{u=1}^{p-1} \frac{1}{|\sin (\pi u / p)|}\right) \cdot k d \sqrt{p} \\
\ll k d p^{1 / 2} \log ^{2 k+1} p .
\end{array}
$$


Therefore

$$
Q_{k}\left(E_{p-1}^{\prime \prime}\right)=\max _{a, b, t, D}\left|\sum_{j=0}^{t} e_{a+j b+d_{1}}^{\prime \prime} \cdots e_{a+j b+d_{k}}^{\prime \prime}\right| \ll k d p^{1 / 2} \log ^{2 k+1} p .
$$

Taking $k=1$ and $d_{1}=0$ in (3.4), we have

$$
W\left(E_{p-1}^{\prime \prime}\right)=\max _{a, b, t}\left|\sum_{j=0}^{t-1} e_{a+j b}^{\prime \prime}\right| \ll d p^{1 / 2} \log ^{3} p .
$$

And taking $a=0, b=1, j=n-1$ and $t=M-1$ in (3.4), we immediately get

$$
C_{k}\left(E_{p-1}^{\prime \prime}\right)=\max _{M, D}\left|\sum_{n=1}^{M} e_{n+d_{1}}^{\prime \prime} \cdots e_{n+d_{k}}^{\prime \prime}\right| \ll k d p^{1 / 2} \log ^{2 k+1} p .
$$

This completes the proof of Theorem 1.2.

Acknowledgments. The author expresses his gratitude to the referee for his/her detailed comments.

\section{References}

[1] J. Cassaigne, S. Ferenczi, C. Mauduit, J. Rivat and A. Sárközy, On finite pseudorandom binary sequences III: The Liouville function, I, Acta Arith. 87 (1999), 367-390.

[2] - , 一, 一, 一, On finite pseudorandom binary sequences IV: The Liouville function, II, ibid. 95 (2000), 343-359.

[3] J. Cassaigne, C. Mauduit and A. Sárközy, On finite pseudorandom binary sequences VII: The measures of pseudorandomness, ibid. 103 (2002), 97-108.

[4] E. Fouvry, P. Michel, J. Rivat and A. Sárközy, On the pseudorandomness of the signs of Kloosterman sums, J. Austral. Math. Soc. 77 (2004), 425-436.

[5] L. Goubin, C. Mauduit and A. Sárközy, Construction of large families of pseudorandom binary sequences, J. Number Theory 106 (2004), 56-69.

[6] K. Gyarmati, On a family of pseudorandom binary sequences, Period. Math. Hungar. 49 (2004), 45-63.

[7] H. N. Liu, New pseudorandom sequences constructed using multiplicative inverses, Acta Arith. 125 (2006), 11-19.

[8] C. Mauduit, J. Rivat and A. Sárközy, Construction of pseudorandom binary sequences using additive characters, Monatsh. Math. 141 (2004), 197-208.

[9] C. Mauduit and A. Sárközy, On finite pseudorandom binary sequences I: Measure of pseudorandomness, the Legendre symbol, Acta Arith. 82 (1997), 365-377.

[10] - - - On the measures of pseudorandomness of binary sequences, Discrete Math. 271 (2003), 195-207.

[11] - - - Construction of pseudorandom binary sequences by using the multiplicative inverse, Acta Math. Hungar. 108 (2005), 239-252.

[12] C. J. Moreno and O. Moreno, Exponential sums and Goppa codes: I, Proc. Amer. Math. Soc. 111 (1991), 523-531. 
[13] A. Sárközy, A finite pseudorandom binary sequence, Studia Sci. Math. Hungar. 38 (2001), 377-384.

[14] W. Zhang, On a problem of P. Gallagher, Acta Math. Hungar. 78 (1998), 345-357.

Department of Mathematics

Northwest University

Xi'an, Shaanxi, P.R. China

E-mail: hnliumath@hotmail.com

Received on 24.11.2006

and in revised form on 19.3.2007 